\title{
Erratum to: Epidermal Growth Factor Receptor (EGFR) Pathway Biomarkers in the Randomized Phase III Trial of Erlotinib Versus Observation in Ovarian Cancer Patients with No Evidence of Disease Progression after First-Line Platinum-Based Chemotherapy
}

\author{
Evelyn Despierre $^{1} \cdot$ Ignace Vergote $^{1} \cdot$ Ryan Anderson $^{2} \cdot$ Corneel Coens $^{3} \cdot$ \\ Dionyssios Katsaros ${ }^{4}$. Fred R. Hirsch ${ }^{2} \cdot$ Bram Boeckx $^{5,6} \cdot$ Marileila Varella-Garcia $^{2}$. \\ Annamaria Ferrero $^{7}$ - Isabelle Ray-Coquard ${ }^{8}$ John A. Green ${ }^{9}$. Christopher Steer ${ }^{10}$. \\ Els M. J. J. Berns ${ }^{11}$ • Antonio Casado ${ }^{12}$ • Diether Lambrechts ${ }^{5,6}$ • Antonio Jimeno ${ }^{2}$
}

Published online: 8 April 2016

(C) Springer International Publishing Switzerland 2016

Erratum to: Targ Oncol (2015) 10:583-596

DOI 10.1007/s11523-015-0369-6

The original version of this article omitted John A. Green and Christopher Steer from the list of authors. The updated list of author names, their affiliations and an updated conflict of interest statement are shown here.

Conflict of Interest Marileila Varella-Garcia is co-inventor on a patent held by the University of Colorado to use

The online version of the original article can be found at http://dx.doi.org/ 10.1007/s11523-015-0369-6.

Evelyn Despierre

evelyndespierre@gmail.com

1 Gynecologic Oncology and Leuven Cancer Institute, and Department of Oncology, KU Leuven, University Hospitals Leuven, Herestraat 49, 3000 Leuven, Belgium

2 University of Colorado Cancer Center, Aurora, Colorado, USA

3 EORTC Headquarters, Brussels, Belgium

4 Azienda Ospedaliera, Presidio Santa Anna, SCDO 3 Ginecologia Oncologica, Università di Torino, Turin, Italy

5 Laboratory for Translational Genetics, Department of Oncology, KU Leuven, Leuven, Belgium

6 Vesalius Research Center (VRC), VIB, Leuven, Belgium
EGFR copy number as biomarker for selection of lung cancer patients for targeted therapy. John A. Green received funding for the sample collection and coordination of the samples in the UK (NCRI) from Cancer Research UK. Evelyn Despierre, Ignace Vergote, Ryan Anderson, Corneel Coens, Dionyssios Katsaros, Fred R. Hirsch, Bram Boeckx, Annamaria Ferrero, Isabelle RayCoquard, Christopher Steer, Els MJJ Berns, Antonio Casado, Diether Lambrechts, and Antonio Jimeno declare no conflict of interest
7 Academic Division of Gynecological Oncology, Mauriziano Hospital, Turin, Italy

8 Département d'Oncologie Médicale Adulte, Centre Leon Bérard, Lyon, France

9 Department of Molecular and Clinical Cancer Medicine, Institute of Translational Medicine, University of Liverpool Clatterbridge Centre for Oncology, Bebington, Merseyside CH63 4JY, United Kingdom

10 Border Medical Oncology, 1/69 Nordsvan Drive, Wodonga, Victoria 3690, Australia

11 Erasmus MC Cancer Institute, Rotterdam, The Netherlands

12 Hospital Universitario Clínico San Carlos, Servicio de Oncologia Medica, Madrid, Spain 